\title{
Ultra-fast intramolecular vibronic coupling revealed by RIXS and RPES maps of an aromatic adsorbate on $\mathrm{TiO}_{2}(110)$
}

\author{
James N. O'Shea, 1 , 2 Karsten Handrup, ${ }^{2}$ Robert H. Temperton, ${ }^{1}$ Andrew J. Gibson, ${ }^{1}$ \\ Alessandro Nicolaou, ${ }^{3}$ Nicolas Jaouen, ${ }^{3}$ John B. Taylor, ${ }^{1}$ Louise C. Mayor, ${ }^{1}$ Janine C. \\ Swarbrick, ${ }^{1}$ and Joachim Schnadt ${ }^{4}$ \\ 1) School of Physics, University of Nottingham, NG7 2RD, \\ $U K$ \\ 2) Department of Physics and Astronomy, Aarhus University, Ny Munkegade 120, \\ DK-8000 Aarhus C, Denmark \\ ${ }^{3)}$ Synchrotron SOLEIL, Saint-Aubin, BP 48, 91192 Gif-sur-Yvette,
} France

4) Division of Synchrotron Radiation Research, Department of Physics, Lund University, Box 118, 22100 Lund, Sweden

Two-dimensional resonant inelastic x-ray scattering (RIXS) and resonant photoelectron spectroscopy (RPES) maps are presented for multilayer and monolayer coverages of an aromatic molecule (bi-isonicotinic acid) on the rutile $\mathrm{TiO}_{2}(110)$ single crystal surface. The data reveals ultra-fast intramolecular vibronic coupling upon core-excitation from the $\mathrm{N} 1 s$ orbital into the lowest unoccupied molecular orbital (LUMO) derived resonance. In the RIXS measurements this results in the splitting of the participator decay channel into a purely elastic line which disperses linearly with excitation energy, and a vibronic coupling channel at constant emission energy. In the RPES measurements the vibronic coupling results in a linear shift in binding energy of the participator channel as the excitation is tuned over the LUMO-derived resonance. Localisation of the vibrations on the molecule on the femtosecond timescale results in predominantly inelastic scattering from the core-excited state in both the physisorbed multilayer and the chemisorbed monolayer.

a)Electronic mail: J.Oshea@nottingham.ac.uk 


\section{INTRODUCTION}

Sensitizing a wide bandgap semiconductor to visible light by the adsorption of a dye molecule lies at the heart of light-harvesting devices such as dye-sensitised solar cells ${ }^{\mathbb{1}}$ and water-splitting photoelectrochemical cells ${ }^{2}$. At these interfaces, a light-harvesting molecule absorbs a photon of visible light through the excitation of an electron from the highestoccupied molecular orbital (HOMO) to the lowest unoccupied molecular orbital (LUMO). Using core level spectroscopies such as resonant photoelectron spectroscopy (RPES) and resonant inelastic x-ray scattering (RIXS) the LUMO is instead populated by the excitation of a core electron through x-ray absorption (XAS). If the electron is localised in the coreexcited LUMO state on the timescale of the core hole lifetime, it can participate in the core hole decay.

In RIXS, the participator (participant ${ }^{3}$ ) channel results in the originally excited electron filling the core hole, and the emission of an x-ray photon of the same energy as the x-ray absorption, resulting in elastic scattering. In RPES, the participator channel can be understood in terms of the originally excited electron filling the core hole, with the concomitant emission of a valence electron in an Auger-like processes. The process leaves the atom (or molecule) in a final state identical to direct photoemission of the occupied valence state, and therefore a resonant enhancement of photoemission at the binding energy of that state. Since the core hole has an intrinsically short lifetime,${ }^{4}$ both of these techniques can be used to probe what happens to the excited electron on the low femtosecond timescale..$^{[5}$ If the electron transfers away from the molecule on the timescale of the core hole lifetime then the core hole decay channel in which this electron is a participant will be depleted. ${ }^{6+10}$

If the electron remains localised in the resonantly excited unoccupied orbital, vibrational effects in the core-excited intermediate state following x-ray absorption can play a role in the core hole decay process. As illustrated in Fig. 1, the initial step in both RIXS and RPES is x-ray absorption and the excitation of the core electron from the ground state into the distribution of vibrational states within the LUMO-derived resonance. By tuning the photon energy over the resonance we can, to some extent, gain control over vibrational excitation of the intermediate state by selectively exciting into specific (higher) vibrational levels. The effect of vibronic coupling is the dissipation of this vibrational energy in the intermediate state on the timescale of the core hole lifetime (6 fs for the $\mathrm{N} 1 s$ core level ${ }^{\sqrt{4}}$ ), resulting in the 
de-excitation step proceeding from a lower vibrational state. The effect of vibronic coupling in RIXS (Fig. 1a) is that the participator channel now contains an inelastic component in addition to the elastic (Rayleigh) scattering. The corresponding effect in RPES (Fig. 1p) is that the energetic driving force for the autoionisation is lowered by the energy difference between the originally excited vibrational state and the lower vibrational state, resulting in a reduction in kinetic energy of the emitted electron by the same amount. Experimentally this would be observed as an apparent shift in binding energy of the participating electrons to higher energy. Evidence for these vibronic effects have previously been observed by RIXS

for $\mathrm{C}_{60}$ multilayers $\frac{1112}{12}$ and aqueous $\mathrm{NH}_{3}, \frac{13}{2}$ and also by RPES for multilayer $\mathrm{C}_{60} \frac{11}{\text {. Here }}$ we compare the effects of vibronic coupling in the N $1 s$ RIXS and RPES maps measured over the over LUMO-derived resonance of bi-isonicotinic acid in the physisorbed multilayer and chemisorbed monolayer on single crystal rutile $\mathrm{TiO}_{2}(110)$. In a recent core-hole clock implementation of RIXS to study the effects of ultra-fast charge transfer from an aromatic molecule (bi-isonicotinic acid) into the conduction band of an oxide surface $\left(\mathrm{TiO}_{2}\right), \frac{14}{14}$ our data suggested that the participator channel was not significantly depleted as it is in the analogous RPES experiment, ${ }^{15}$ while strong evidence for ultra-fast vibronic coupling was observed over the LUMO-derived resonance. To further understand the similarities between these two techniques, here we directly compare the effects of vibronic coupling in the N $1 s$ RIXS and RPES maps measured over the LUMO-derived resonance of bi-isonicotinic acid in the physisorbed multilayer and chemisorbed monolayer on the single crystal rutile $\mathrm{TiO}_{2}(110)$ surface.

\section{METHOD}

RIXS measurements were performed at the AERHA spectrometer ${ }^{16}$ at the SEXTANTS beamline at Synchrotron SOLEIL. 17 Samples were prepared in a separate UHV system in our home laboratory equipped with a Scienta R3000 analyser and dual anode (Al k $\alpha, \mathrm{Mg}$ $\mathrm{k} \alpha$ ) x-ray source. Substrates were rutile $\mathrm{TiO}_{2}(110)$ single crystals (Pi-Kem, UK) cleaned by repeated cycles of sputtering at $2 \mathrm{kV} \mathrm{Ar}^{+}$followed by $1 \mathrm{kV} \mathrm{Ar}^{+}$, and annealing in UHV to $875 \mathrm{~K}$ until no C $1 s$ signal was observed by x-ray photoelectron spectroscopy (XPS). Multilayers of bi-isonicotinic acid (4,4-dicarboxy-2,2-bipyridine from Alfa Aesar), were deposited 


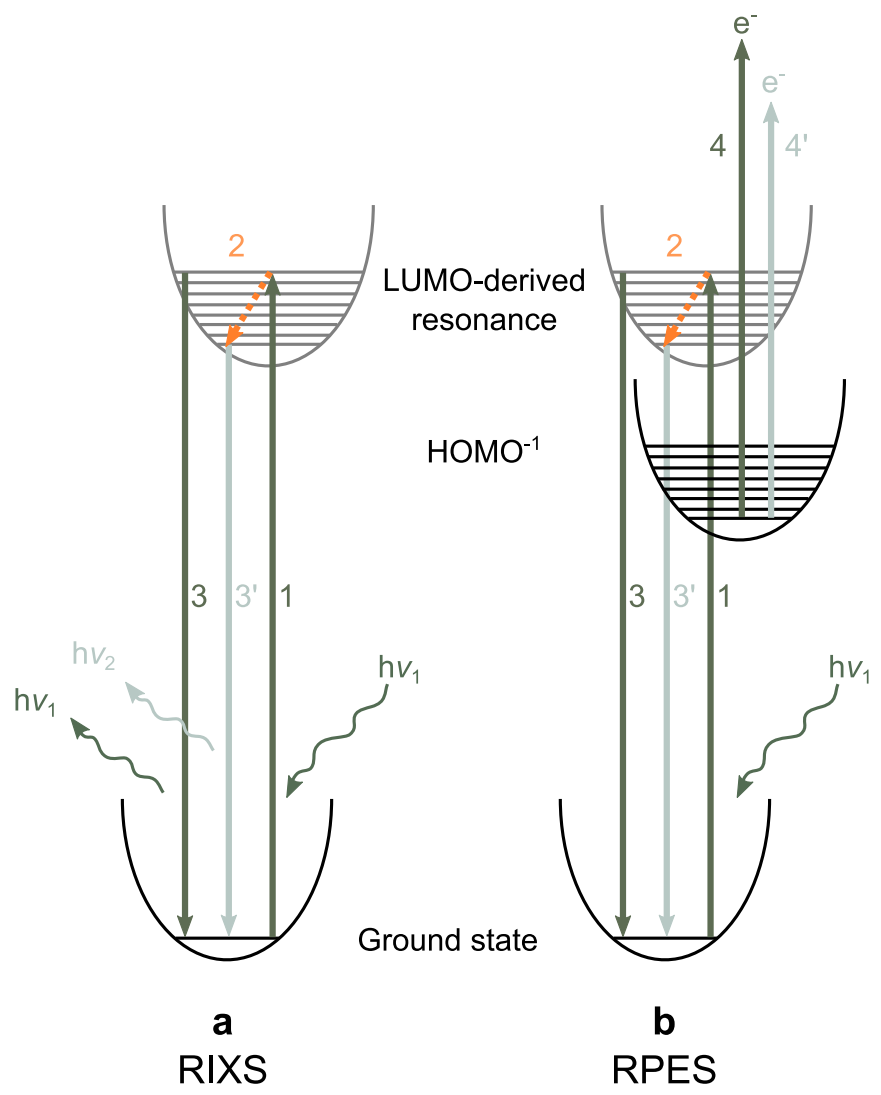

FIG. 1. Schematic of the electronic transitions in the presence and absence of vibronic coupling in a simplified Franck-Condon picture of a) RIXS and b) RPES. Transition 1 is the x-ray absorption event from the N $1 s$ core level in the ground state to a specific vibrational state of the LUMOderived resonance. Transition 3 is the elastic participator event which leads to an elastic photon $\left(\mathrm{h} \nu_{1}\right)$ in RIXS and the emission of an electron (transition 4) in RPES. Transition 2 represents the dissipation of vibrational energy within the core hole lifetime leading to the inelastic participator decay shown by transition 3'. In RIXS this leads to the emission of a photon $\left(\mathrm{h} \nu_{2}\right)$ of lower energy (inelastic scattering) and in RPES to the emission of an electron with a lower kinetic energy (transition 4'). While not relevant to the present discussion, it is also possible to end up in a state other than the ground state in RIXS and RPES (omitted for clarity).

onto the prepared substrates held at room temperature by sublimation from a Knudsen-type cell evaporation source until no Ti $2 p$ signal was detected in the XP spectrum. The multilayer samples were then transported to SOLEIL and transferred into the analysis chamber of the SEXTANTS beamline where the base pressure was $1 \times 10^{-9}$ mbar. For RIXS measurements on the multilayers, samples were used as prepared. For RIXS measurements of the 
monolayers, these were prepared by annealing fresh multilayer samples in situ to $500 \mathrm{~K}$ to desorb the physisorbed multilayer to leave just the chemisorbed monolayer. The thickness of the multilayer films is estimated from XPS to be around $10 \mathrm{~nm}$. Due to the increased penetration depth of x-rays compared to photoelectrons, there will be a small contribution of the first monolayer in the multilayer RIXS measurements. However, this is estimated to be at most a few percent of the overall signal.

The incident beam at SEXTANTS is focused to a spot size of $2(\mathrm{v}) \mu \mathrm{m} \times 100(\mathrm{~h}) \mu \mathrm{m}$. To prevent beam damage of the molecules, the sample was continuously moved during the measurements at a rate that was shown to not give rise to changes between successive fast XAS scans. Due to the very low x-ray emission signal monolayer N $1 s$ RIXS maps were accumulated over more than one sample and the total x-ray emission intensity detected at each absorption energy normalised to the corresponding fluorescence-yield XAS intensity measured using an MCP detector. The photon energy scale of the RIXS spectrometer was calibrated by measuring the elastic peak at $5 \mathrm{eV}$ increments across the whole detector range using linear vertical polarisation of the incoming beam. In the measurement of the RIXS maps and XAS over the N $1 s$ absorption edge the beam was linearly polarised in the horizontal plane (in the plane of the spectrometer), and the incidence angle was $35^{\circ}$ with respect to the surface. The overall measurement resolution was set to $200 \mathrm{meV}$ (compared to the optimum resolution of $110 \mathrm{meV}$ ) as a compromise between energy resolution and spectrometer transmission to keep the adsorbed molecule below the radiation damage threshold. For the multilayer RIXS map, each line is an x-ray emission spectrum accumulated for 10 mins at $0.1 \mathrm{eV}$ intervals. For the monolayer RIXS map each line is the average of between 3 and 12 spectra accumulated for 10 mins, at $0.2 \mathrm{eV}$ intervals.

The RPES measurements were performed at the I311 beamline ${ }^{18}$ on the MAX-II ring of the MAX IV Laboratory synchrotron radiation facility, equipped with an upgraded Scienta SES200 hemispherical analyser. The base pressure in the analysis chamber was in the mid $10^{-11}$ mbar range, and that in the preparation chamber was in the low $10^{-10}$ mbar range. The rutile $\mathrm{TiO}_{2}(110)$ crystal was mounted on a Ta back-plate and a thermocouple attached close to the crystal, and prepared in the same way as in the RIXS experiments. Bi-isonicotinic acid was evaporated in situ onto the cleaned crystal in the preparation chamber of the beamline in the same way as described for the RIXS experiments. To prevent beam damage, spectra were recorded while the sample was swept across the beam, ensuring the motion was in the 
focal plane of the analyzer. Exit slits of the monochromator were set to give a resolution of $100 \mathrm{meV}$ for photons of energy $\mathrm{h} \nu=400 \mathrm{eV}$. The photon energy was calibrated from the energy separation of the C $1 s$ core level photoemission peaks excited by x-rays from the first-order and second-order transmission of the beamline's monochromator. The binding energy scale of the RPES datasets are left uncalibrated as no reliable reference point was available for the multilayer and only relative shifts are required for a full interpretation of the data.

\section{RESULTS AND DISCUSSION}

The multilayer film $(\sim 10 \mathrm{~nm})$ consists of many layers of physisorbed bi-isonicotinic acid of the form shown in Fig. 2a, effectively isolated from the underlying substrate. The monolayer film, on the other hand, is chemisorbed to the rutile $\mathrm{TiO}_{2}(110)$ surface in a $2 \mathrm{M}$-bidentate bonding configuration mediated by the deprotonation of the carboxyl groups (shown in Fig. 2 b) and the subsequent bonding of the carboxylate oxygen atoms to the titanium atoms of the oxide surface. The RIXS map measured over the absorption energy range corresponding to excitation of $\mathrm{N} 1 s$ core electrons into the LUMO-derived resonance for a multilayer and a monolayer of bi-isonicotinic acid on $\mathrm{TiO}_{2}(110)$ is shown in Figs. 2 c and e, respectively. Also shown are line profile spectra measured at the LUMO-derived resonance, $0.2 \mathrm{eV}$ above, and $0.4 \mathrm{eV}$ above the resonance in Figs. $2 \mathrm{~d}$ and $\mathrm{f}$. The horizontal axis of the map represents the x-ray emission energy, while the vertical axis represents the $\mathrm{x}$-ray absorption energy. Features below around $396 \mathrm{eV}$ emission energy are attributed to inelastic x-ray scattering arising from the $\mathrm{N} 1 s$ core hole being filled by valence electrons from the occupied molecular orbitals. This is the projection of the occupied densities of states of the molecule onto the nitrogen core level governed by the appropriate dipole selection rule (these transitions are omitted in Fig. 1 for clarity). Above $396 \mathrm{eV}$ emission energy we observe the participator decay features. The narrow diagonal feature observed on the high emission energy side is the purely elastic line, where the emission energy is equal to the absorption energy, corresponding to transition 3 in Fig. 1 a.

In the RIXS map of both the multilayer (Fig. 22) and monolayer (Fig. 2p) there is substantial intensity on the low emission energy side of the elastic line as the absorption is tuned over the LUMO resonance. This is easiest to analyse for the multilayer spectrum 

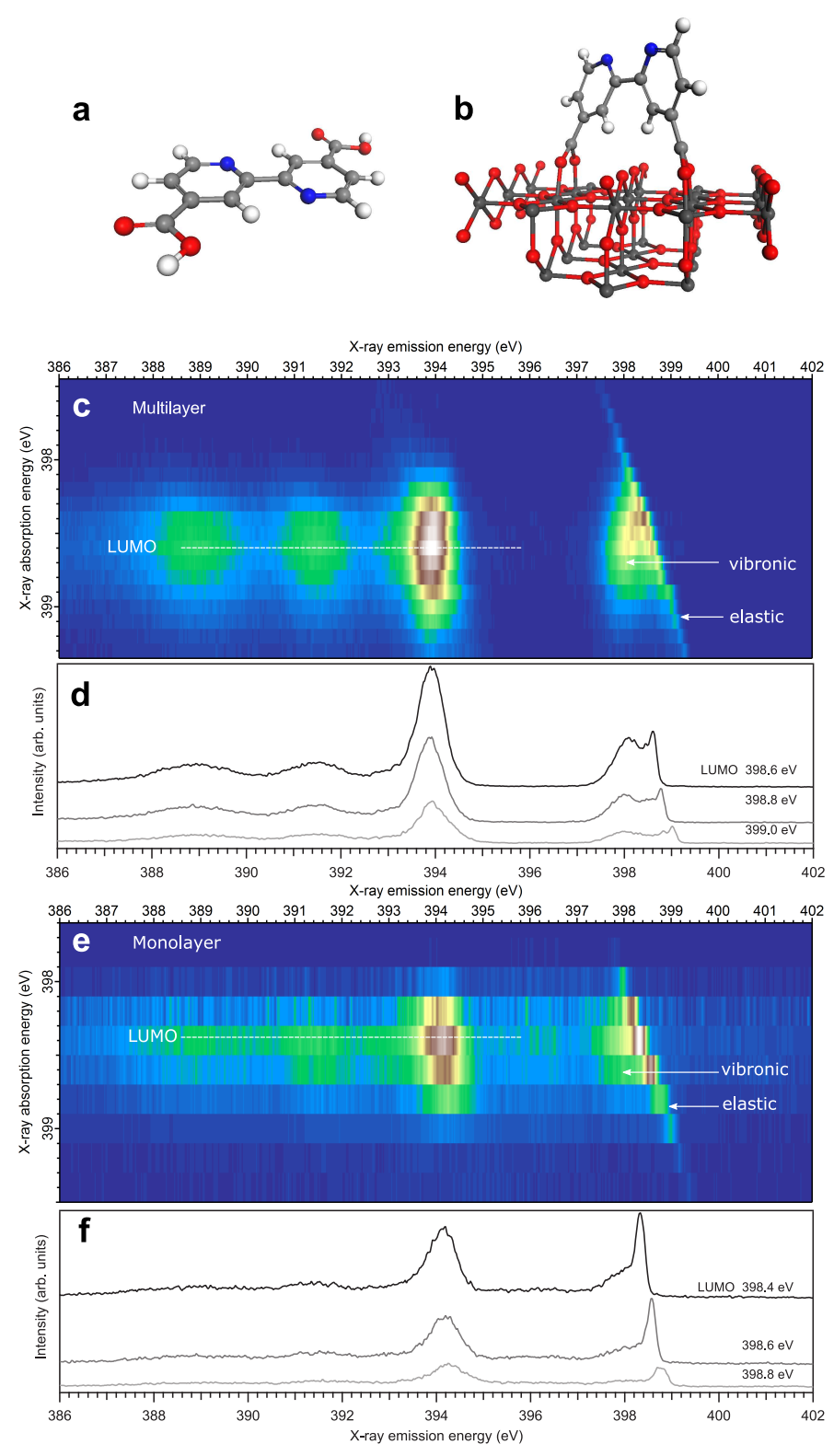

FIG. 2. Molecular structure of a) an isolated bi-isonicotinic acid molecule an b) bi-isonicotinic acid chemisorbed in a $2 \mathrm{M}$-bidentate configuration on the rutile $\mathrm{TiO}_{2}(110)$ single crystal surface mediated through the deprotonated carboxyl groups. N $1 s$ RIXS maps for c) physisorbed biisonicotinic acid multilayer and e) chemisorbed monolayer on the rutile $\mathrm{TiO}_{2}(110)$ single crystal surface. Single energy RIXS spectra extracted from the RIXS maps at the specified excitation energies for the d) multilayer and f) monolayer. The experimental data is a subset of the data from our previously published RIXS results, rescaled to focus on the participator region of the LUMO-derived resonance. 14 
(Fig. 26) due to the much stronger signal arising from the increased number of molecules being probed. This feature has an intensity centred around an (almost) constant emission energy of $398 \mathrm{eV}$, also seen in the line profiles in Fig. 2d. This corresponds to participator decay always from the same vibrational component of the intermediate state (transition 3' in Fig. 17). Although the excitation is tuned to excite increasingly energetic vibrational components within the LUMO resonance, dissipation of the vibrational energy in the coreexcited state (transition 2 in Fig. 17) results in the participator decay proceeding from the lowest accessible state. Thus, a substantial component of the x-ray emission signal in the participator region of the RIXS appears at an almost constant emission energy as the excitation energy is increased.

The RIXS signal from the monolayer (Fig. 2e) is much weaker than that from the multilayer due to the much lower number of atoms present. Nevertheless, the same features are clearly present for the monolayer as discussed for the multilayer. The splitting of the vibronic coupling line from the elastic line in the participator channel is more difficult to resolve due to the relative increase in the pure elastic intensity (compared to the multilayer). However, the broadening is consistent with the presence of the vibronic channel and constant energy of this feature is observed in the line profiles shown in Fig. 2f. The measurement statistics for the monolayer are insufficient to analyse the shape of the envelope of the vibrational structure in the vibronic region. However, we can conclude that while coupling the molecule to the oxide surface could, in principle, modify the energies of the core-excited vibrational states, the presence of vibronic coupling on the timescale of the core hole lifetime is still clearly observed. In both cases the originally excited electron is required to be localised in the LUMO on the femtosecond timescale. This is entirely consistent with previous results for this molecule-surface system: molecules in the physisorbed multilayer are decoupled from the surface so there is nowhere for the excited electron to tunnel into. In addition, the LUMO-derived resonance is an excitonic state, lowered in energy such that it lies within the fundamental gap of the molecule, isolating it from empty states in the surrounding molecules. This same excitonic effect in the monolayer leads to the LUMO resonance being pulled down below the conduction band edge of the titanium dioxide such that it also has no overlap with empty density of states even in the coupled monolayer!15

The N $1 s$ RPES measurements over the LUMO-derived resonance of the bi-isonicotinic acid multilayer and monolayer adsorbed on $\mathrm{TiO}_{2}(110)$ are shown in Figs. $3 \mathrm{a}$ and 3b, re- 


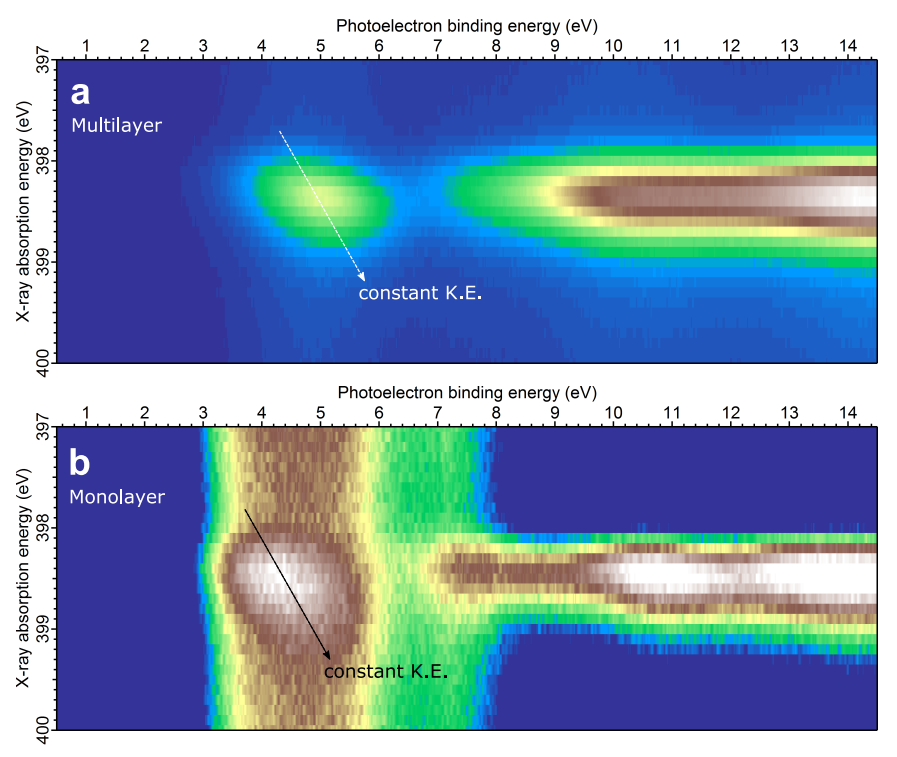

FIG. 3. N $1 s$ RPES maps for a) a physisorbed bi-isonicotinic acid multilayer and b) the chemisorbed monolayer on the rutile $\mathrm{TiO}_{2}(110)$ single crystal surface. In both spectra a line of constant kinetic energy is added as a guide to show that the participator resonant photoemission signal from the LUMO-Core-HOMO decay channel (see figure 1 $\mathrm{b}$ ) tracks with constant kinetic energy due to vibronic coupling.

spectively. Here, as in the case of the RIXS data, the same features are observed for both the monolayer and multilayers and can therefore be discussed together. The participator channel in RPES is the resonant enhancement of photoemission from the occupied valence state involved in the core hole decay. In this case, an electron from the core-excited LUMO fills the core hole and an electron from the highest occupied molecular orbital (HOMO) is emitted in an Auger-like process. This is schematically illustrated in Fig. 1b. The participator channel is observed in the spectra in Fig. 3 as a 'twisted' elliptical feature centred around $5 \mathrm{eV}$ binding energy for the multilayer and $4.5 \mathrm{eV}$ for the monolayer. This 'twist' observed for this feature in both cases can be attributed to the vibronic coupling already discussed for RIXS.

In the absence of vibronic coupling the binding energy of the participator channel is expected to remain constant as the absorption energy is increased. The molecule is left in the same final state as direct photoemission from the HOMO and therefore should always have the binding energy of the HOMO. In terms of the transitions illustrated in Fig. 1 b, participator decay involving a higher vibrational state of the LUMO (transition 3) will re- 
sult in a correspondingly energetic electron being emitted (transition 4) and so the kinetic energy of the measured electrons will increase linearly with photon energy, thus having a constant binding energy. On the other hand, if through vibronic coupling (transition 2), the participator decay proceeds from (almost) the same vibrational state of the LUMO-derived resonance (transition 3'), the kinetic energy of the emitted electron will be correspondingly lower (transition 4'). In an Auger picture this can be understood as the three energy levels involved in the process always being the same, thus resulting in electron emission of constant kinetic energy. On the binding energy scale of Fig. 3 the participator signal disperses linearly with the expected slope for electrons with constant kinetic energy over the LUMO resonance for both the multilayer and monolayer, consistent with the vibronic coupling scheme illustrated in Fig. 1 and observed in the RIXS data of Fig. 2, The features observed to higher binding energy in Fig. 3 (above $7 \mathrm{eV}$ ) comprise a mixture of resonant photoemission and resonant Auger. In principle, the resonant photoemission peaks arising from participator decay from the HOMO-1 and HOMO-2 in this region might also be twisted due to vibronic coupling effects. However, these features overlap with the intense resonant Auger signal arising from the spectator process. Here the Auger decay proceeds via electrons located in the highest occupied molecular orbitals while the effect of the electron excited to the LUMO is simply to increase the kinetic energy slightly of the emitted electron by the so-called spectator shift. ${ }^{5}$ Spectator electrons disperse with constant kinetic energy and therefore prevent an analysis of the vibronic effects in any overlapping resonant photoemission. In the case of bi-isonicotinic acid, the participator and spectator signals do not overlap in the region of the HOMO as they are well-separated in energy due to the large HOMO-LUMO gap of the molecule. 19

\section{CONCLUSIONS}

Resonant inelastic x-ray scattering (RIXS) and resonant photoelectron spectroscopy (RPES) maps measured over the $\mathrm{N} 1 s$ absorption edge for resonant excitation into the LUMO of the aromatic molecule bi-isonicotinic acid have been used to explore the effects of vibronic coupling on the de-excitation of the core-excited state. We find that the vibra- 
tions excited in the intermediate state are decoupled from the de-excitation process on the timescale of the core hole lifetime of a few femtoseconds and modify the energetics of the participator decay channel. In RIXS this leads to predominantly inelastic scattering with an almost constant emission energy due to transitions proceeding form the same vibrational state as the photon energy is tuned through the LUMO resonance. In RPES, the same process leads to a resonant photoemission participator feature with a constant kinetic energy rather than the expected constant binding energy. Since the coupling between neighbouring molecules in the multilayer is weak, the vibrations must be both localised to and delocalised within the probed molecule on the low femtosecond timescale. From the similarity of the spectra for the multilayer and monolayer we conclude that the same process occurs in both. It is worth noting that bi-isonicotinic acid is a significantly smaller molecule than $\mathrm{C}_{60}$, for which the same process has previously been observed in the molecular solid. This raises the question of how small a molecule can be for which we can still observe the dispersion of the vibrations on the molecule within the probed timescale. The agreement between RIXS and RPES in terms of the vibronic coupling suggests that ultra-fast processes occurring during the intermediate core-excited state should be equally observable in both techniques, further suggesting that a core-hole clock implementation of RIXS in which the participator channel is depleted by ultra-fast charge transfer away from the molecule should be feasible. Experiments are currently planned to explore this process in other molecule-surface systems.

\section{ACKNOWLEDGEMENTS}

Funding was provided by RCUK — Engineering and Physical Sciences Research Council (EPSRC) and Molecularspray Ltd through a DTG/CASE conversion studentship, and the European Community - Research Infrastructure Action under the FP6 "Structuring the European Research Area" Programme (through the Integrated Infrastructure Initiative "Integrating Activity on Synchrotron and Free Electron Laser Science").

\section{REFERENCES}

${ }^{1}$ M. Grätzel, J. Photochem. Photobiol. C 4, 145 (2003). 
${ }^{2}$ J. J. Concepcion, J. W. Jurss, M. K. Brennaman, P. G. Hoertz, A. O. T. Patrocinio, N. Y. Murakami Iha, J. L. Templeton, and T. J. Meyer, Acc. Chem. Res. 42, 1954 (2009).

${ }^{3}$ Participator has become the accepted term in the literature to describe the core-hole decay process in which the originally excited electron is a direct participant, resulting in resonant photoemission in RPES and inelastic scattering in RIXS. In Ref. 5 by P. A. Brühwiler et al, the grammatically correct term participant is used instead. Both terms refer to the same processes.

${ }^{4}$ B. Kempgens, A. Kivimäki, M. Neeb, H. M. Köppe, A. M. Bradshaw, and J. Feldhaus, J. Phys. Chem. B 29, 5389 (1996).

${ }^{5}$ P. A. Brühwiler, O. Karis, and N. Mårtensson, Rev. Mod. Phys. 74, 703 (2002).

${ }^{6}$ J. B. Taylor, L. C. Mayor, J. C. Swarbrick, J. N. OShea, and J. Schnadt, J. Phys. Chem. C 111, 16646 (2007).

${ }^{7}$ L. C. Mayor, J. B. Taylor, G. Magnano, A. Rienzo, C. J. Satterley, J. N. O'Shea, and J. Schnadt, J. Chem. Phys. 129, 114701 (2008).

${ }^{8}$ M. Weston, A. J. Britton, and J. N. O'Shea, J. Chem. Phys 134, 054705 (2011).

${ }^{9}$ M. Weston, K. Handrup, T. J. Reade, N. R. Champness, and J. N. O'Shea, J. Chem. Phys. 137, 224706 (2012).

${ }^{10}$ A. J. Britton, M. Weston, and J. N. O'Shea, Phys. Rev. Letters 109, 017401 (2012).

${ }^{11}$ L. Kjeldgaard, T. Kaambre, J. Schiessling, I. Marenne, J. O'Shea, J. Schnadt, C. Glover, M. Nagasono, D. Nordlund, M. Garnier, L. Qian, J. Rubensson, P. Rudolf, N. Martensson, J. Nordgren, and P. Bruhwiler, Phys. Rev. B 72, 205414 (2005).

${ }^{12}$ L. Weinhardt, O. Fuchs, D. Batchelor, M. Baer, M. Blum, J. D. Denlinger, W. Yang, A. Schoell, F. Reinert, E. Umbach, and C. Heske, J. Chem. Phys. 135, 104705 (2011).

${ }^{13}$ L. Weinhardt, E. Ertan, M. Iannuzzi, M. Weigand, O. Fuchs, M. Bar, M. Blum, J. D. Denlinger, W. Yang, E. Umbach, M. Odelius, and C. Heske, Phys. Chem. Chem. Phys. 17,27145 (2015).

${ }^{14}$ J. N. O'Shea, K. Handrup, R. H. Temperton, A. J. Gibson, A. Nicolaou, and N. Jaouen, J. Chem. Phys. 147, 134705 (2017).

${ }^{15}$ J. Schnadt, P. A. Brühwiler, L. Patthey, J. N. O'Shea, S. Södergren, M. Odelius, R. Ahuja, O. Karis, M. Bässler, P. Persson, H. Siegbahn, S. Lunell, and N. Mårtensson, Nature 418, 620 (2002). 
${ }^{16}$ S. G. Chiuzbaian, C. F. Hague, A. Avila, R. Delaunay, N. Jaouen, M. Sacchi, F. Polack, M. Thomasset, B. Lagarde, A. Nicolaou, S. Brignolo, C. Baumier, J. Luening, and J.-M. Mariot, Rev. Sci. Instrum. 85, 043108 (2014).

${ }^{17}$ M. Sacchi, N. Jaouen, H. Popescu, R. Gaudemer, J. M. Tonnerre, S. G. Chiuzbaian, C. F. Hague, A. Delmotte, J. M. Dubuisson, G. Cauchon, B. Lagarde, and F. Polack, in 11th International Conference on Synchrotron Radiation Instrumentation (SRI 2012), J. Phys. Conf. Ser., Vol. 425, edited by Susini, J and Dumas, P (2013) p. 072018.

${ }^{18}$ R. Nyholm, J. N. Andersen, U. Johansson, B. N. Jensen, and I. Lindau, Nucl. Instrum. Meth. 467, 520 (2001).

${ }^{19}$ R. H. Temperton, A. J. Gibson, K. Handrup, and J. N. O'Shea, J. Chem. Phys. 147, 054703 (2017). 